\title{
The Social Support of Left-behind Children's Growth Education
}

\author{
Heting Sun \\ Politics and Administration \\ Wuhan University of Technology \\ Wuhan 430063, China; \\ 569542267@qq.com
}

\begin{abstract}
Based on the existing research at home and abroad, this essay adopts the theory of social support and does a research with the help of questionnaires. It objectively classifies the factors which will affect the growth of Home-parked Children in rural areas into four types: parental support, teacher support, peer support, and organizational support. Besides, the essay explains and explores the effect and relevant measures in respect of the four factors to improve the social support net for Home-parked Children and search more targeted ways to make a better growth environment to make them grow healthily.
\end{abstract}

Keywords-Home-parked Children, social support

\section{INTRODUCTION}

Left-behind children, namely rural left-behind children, refer to the minors at the age ranging from 6 to 16 and raised, taken care of and managed by parent, elders or others or lived independently by themselves in the rural areas because their parents or either of them has to go out for a long time. As shown in the Research Report on the Situation of Rural Left-behind Children in China released by the All-China Women's Federation in 2014, there was 61.0255 million rural left-behind children nationwide, accounting for $37.7 \%$ of the rural stay-at-home children and $21.88 \%$ of the total children in China. At the same time, it was demonstrated in the report that there were 58 million left-behind children in rural areas of China in 2008, compared with the increased 5.2\% in 2014. It has indicated the large-scale growth of left-behind children in China's rural areas. The increasing children left behind in the rural areas of China arise from the unbalanced economic development and the changing environment both internal and external is making influences on their growth and development. In spite of the society's great concerned for their future, the actual operation not deserves to be promoted. The paper is designed to establish a more practical supporting response system to create conditions for the healthy development of left-behind children in rural areas via strengthening the psychological dynamic comparative study of left-behind children and making up for the deficiencies existing in the strategy for the dynamic development of left-behind children. Additionally, the paper helps to further increase the attention of the society to the left-behind children in rural areas and alleviate the stigmatization of the society for left behind children. And it also integrates the social resources under the collaboration and support of society and government, improves the social support network for its growth needs and further puts forward more targeted methods for a more harmonious social atmosphere in which they can grow up healthily.

In the paper, the social support theory, namely one selective social behavior that the certain social network is to help the weak in society in the material and spiritual manners, is mainly adopted. Based on the existing research at home and abroad, the social support system for the total sample of 4650 rural left-behind children has been investigated through the research method of questionnaire in the paper, as shown in the Table 1.

TABLE I. SAMPLE SELECTION OF LEFT-BEHIND CHILDREN AND NON-LEFT-BEHIND CHILDREN

\begin{tabular}{ccc}
\hline & Frequency & Percentage \\
\hline $\begin{array}{c}\text { Left-behind Children } \\
\text { Non-left-behind } \\
\text { Children } \\
\text { Total }\end{array}$ & 3127 & 67.25 \\
& 1523 & 32.75 \\
\hline
\end{tabular}

\section{ANALYSIS}

After the data checking, eigenvalues and eigenvectors of the collected questionnaires, there are four factors which are separately the children's parents support, teacher support, peer support and organization support extracted by the principal component factors, with the details as follows:

\section{A. Rural left-behind children are more supported by their parents in terms of family care}

As shown in the Fig 1 and Fig 2, apart from the different parental supports between left-behind children and non-left behind children, the comparison also implies that more left-behind children believe that they are loved by their parents than those thinking that they have a good relationship with their parents. Accordingly, it can be concluded that the care provided by the parents is the main part of family cares which involves more than just parents. And the distinct differences between the proportion that the left-behind children believing they are highly hated by the families and that of keeping a bad relationship with their parents indicate that the indispensable parental support constitutes the main part of family care for the stay-at-home children living in the areas in the case of absent family care and support. 


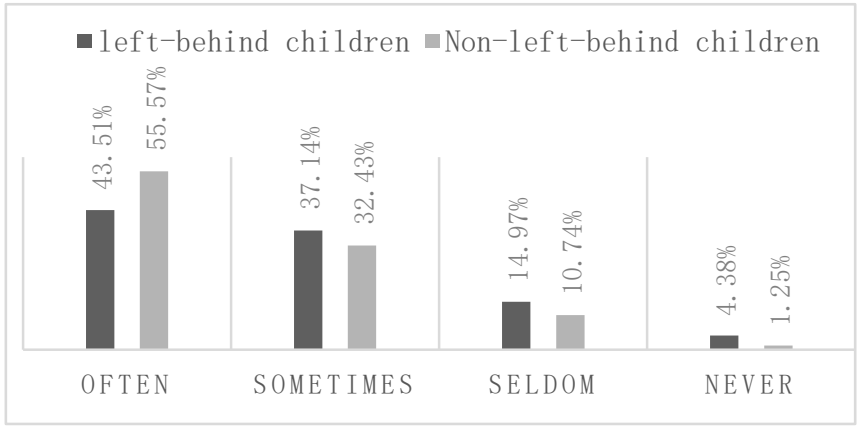

Fig. 1. The frequency of chatting with family

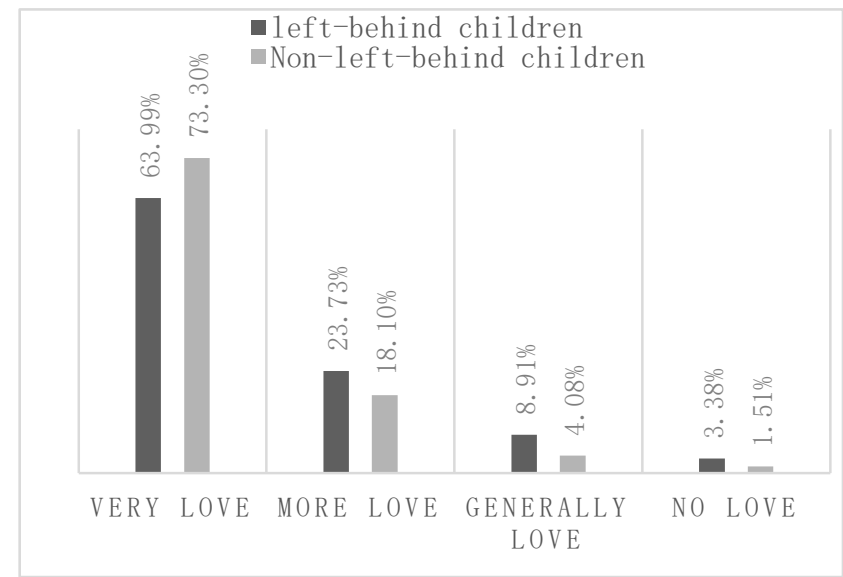

Fig. 2. The degree of family love

B. Rural left-behind children are more expected to get support from teachers in life

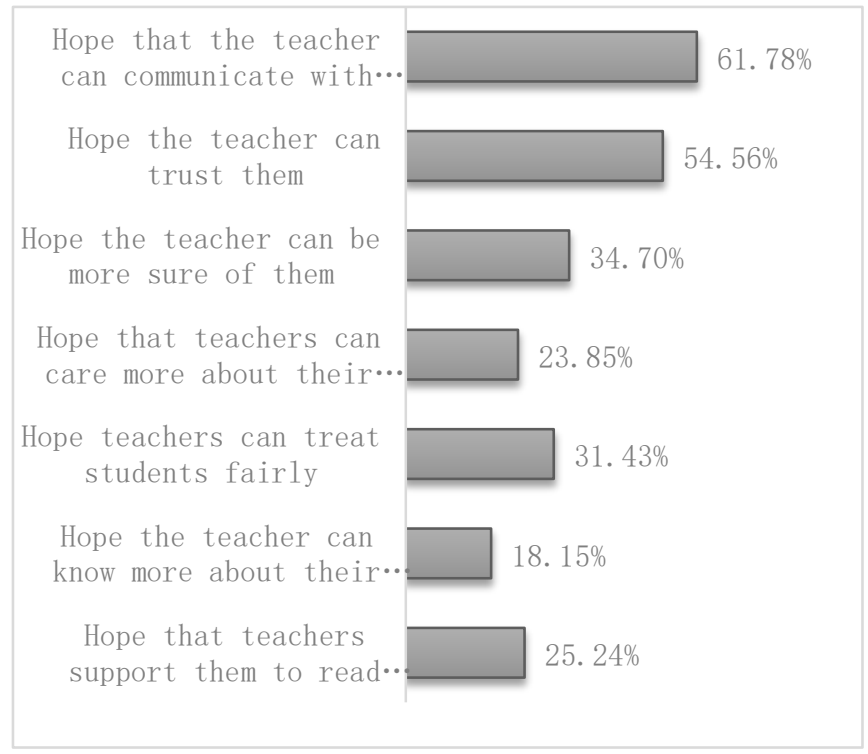

Fig. 3. Teachers' support for left behind children in rural areas

As shown in the Fig 3, the teacher's concern about the life of rural left-behind children seems particularly important in the case of the absent parental care for the life of their children when taking the parents' going out into consideration. In this regard, the rural left-behind children are more expected to get support from teachers in life.

\section{The peer group has greater influences on the rural left-behind children}

During the investigation of the factors affecting the left-behind children in rural areas, there are 1457 people selecting the element of classmates and friends, accounting for $30.97 \%$ of the sample. When being asked how they would be affected by their parents' out-migrating for work, 325 rural left behind children said they would feel free, which accounted for $10.49 \%$. From this, we can know that the elements of classmates and friends mainly affects the left-behind children, while the inducement from their classmates and friends makes them feel free for their parents' going out for work. In this regard, it is believed that the peer group has greater influences on the children left behind the rural areas when it comes to what are they affected.

\section{Rural left-behind children are more inclined to the help from school than social workers}

It is shown in the survey about whom the rural left behind children will turn for help in the face of difficulties that 700 rural left behind children who will turn to schools account for $14.73 \%$ while 528 of them will turn to social workers. It can be seen that the rural left behind children are more likely to turn to the help from the school when they are in trouble.

\section{DISCUSSION}

From the descriptive analysis above, it can be concluded that the support situation of rural left-behind children is generally reflected in four aspects, including the parental support, teachers' support, peer support and organizational support of left-behind children in rural areas. It is specifically discussed as follows:

\section{A. Left-behind children need more emotional support from their parents}

Family plays an indispensable role in the initiation of children's growth, and the parental support makes certain influences on the character development and growth track of their children. It can be easily seen from the parents' support mentioned above that the family supports of left-behind children mostly consist of the parental support, indicating that the supports provided by the parents mean a lot to the healthy growth of the left-behind children. And their speech and deportment, attitudes and the behavioral style of stability when educating the kids will greatly affect the socialization process of children. We have realized in the investigation that the parents are more concerned about their study but less involved in the emotional state and psychological problems of their children, making it unlikely for them to get comfort from parents' companionship and communication as well as release and resolve the negative emotions and psychological problems. Consequently, the various problems like weak personality, inferiority, loneliness, low learning ability, lack of life care and difficulty in integrating into society will come.

The parents of left behind children shall change their ideas, care more about their life and emotion and provide more emotional comfort and support to their children, instead of 
merely focusing on children's learning. In addition, the parents shall uphold the appropriate education responsibility philosophy of "Father shall be blamed for the no-teaching of son", and handle the relationship between going out for earning money and educating their children in a proper way. At the same time, the communication with school teachers and class teachers shall be strengthened. In spite of working outsides, they shall shoulder the responsibility of educating children and keep active contact with the class teachers and head teacher to understand the development and change of kids and jointly discuss the strategies and methods for education.

\section{B. Left-behind children more long for the teacher's attention to their life}

School is the main place for left behind children to study and live. In the case of insufficient parental supports, the support provided by the teachers occupying the pedestal as the children worship idols and models plays an irreplaceable role in the healthy growth of the left-behind children while making up for the lacking parental support emotionally. It is demonstrated in the several variables mentioned above that the left-behind children more long for the teacher's attention to their living conditions and family, and more children without living with their parents express that the study is never instructed and life is unattended with respect to the influence brought by the parents' going out. So it can be known that the left behind children are more eager to get the teacher's attention to their lives from the aspect of teachers' support. And the shortage of teachers' basic concern for the children, less attention to the after-class life, psychological problems and family situation, missing contact with the left-behind children and their parents as well as the negligence of their psychological appeal will trigger the absence of teachers' support for the left-behind children and also will breed and indulge the studying weariness of the children to some extent.

Therefore, teachers should not only provide rural children with learning help but also the supportive behavior, making them feel the love and understanding from the teacher. And the teachers shall also strengthen the communication with rural children and help them in addressing the problems existing in learning, life and psychology. At the same time, it shall ease the negative emotions of the rural children timely, guide them to deal with the problem in a correct way and attitude and provide them with a good psychological environment and school sphere. Moreover, it is expected to actively undertake the social responsibility, contact and remind guardians of the strengthened guardianship, timely report the school drop-outs in a written form to the relevant departments and assist in enhancing the emotional connection between left-behind children and their parents, thus maintaining the continuity and stability of school educational care measures.

\section{Effect of peer group can meet the sense of freedom of left-behind children}

The peer support of the rural children mainly consists of the supports from friends and classmates. The children and adolescents are more likely to be affected by the external environment because of their viewing the problem in the one-sided and emotional way and their weak self-control ability arising from unstable emotion and psychology. Coupled with the transition period from juvenile to adult, namely the "weaning period" at the psychological level, the adolescents are psychologically distinguished by imitation, rebellion and pursuit of freedom frequently. In the data above, the opinion that the parents' going out make the children feel free score highest, which illustrates that the parents' long-term absence and weak family constraints owing to out-migration for work change the left-behind children's personality and value judgments to a certain extent, making them feel free from the parents' going out. So it can be seen that peer group makes more influences on meeting the children's sense of freedom. They are more easily affected by peer groups while feeing free. Apart from the effect on the mainstream culture of society brought by the positive group, the weakness of family control makes them more easily exposed to the influenced of the culture transmitted by negative groups, thus obstructing their healthy growth and the shaping of correct values. Accordingly, it is of great significance to pay attention to the exchange process between the rural children and their peers, set up the guiding mechanism fitting the development, promote the benign development of rural children's peer interaction and accelerate the increasingly improved and mature outlook on life and values of children and adolescents.

\section{Left-behind children are more willing to turn to school or social workers for help}

As the cause of the current situation of left-behind children, social support is also an effective way to solve this status quo. According to the survey, the left-behind children are more willing to turn to the school and social workers when facing difficulties than the non-left behind children. The social work professional service institutions play a positive role in the constructing the rural children's social support, integrating more abundant resources for the children, providing more support services, taking the professional advantages and promoting the specialized rural children's services. And the school, as the important place for the left-behind children's school life, also provides certain emotional support at different level for children while instilling the knowledge to them. Additionally, the normality and reliability of the school make them more likely to turn to school or social workers in the face of difficulties.

The linkage between social work institutions and schools for rural children are mainly embodied in the following aspects. On the one hand, social work can strive for the resources support from the government and society. On the other hand, directly conducting the work in the school, the social work can incorporate the school teachers, left-behind children and other children into the objects to be served, intervene in the different groups diversely and jointly create a good environment for the left-behind children's learning and growth. For example, the school teachers can be educated, the full-time teachers can be cultivated and the perfect record of children left behind shall be established by the school. The specialized organizations involved in the left-behind children care shall be set up for service and it shall also make the best of the peer groups playing a positive role. In addition, the social work institution can take the initiative to undertake the government's projects related to left-behind children to make up for the lack of 
government concern for the left-behind children. And it can also promote the specialized left-behind children services for the support problems, warn and prevent the problems related to the left-behind children services and provide the professional decision-making consulting and suggestions.

\section{CONCLUSION}

For the children at a disadvantage of society, social support is an effective way to help them get out of predicament, share the achievements of social prosperity, develop in the round and realize social justice. In this regard, it plays a crucial role in pushing forward their healthy and happy growth by increasingly improving and strengthening the social support of different objects on the rural left-behind children, creating the better living environment for the children left in the rural areas and providing them with the sufficient and effective development resources, based on the current problems and the actual condition.

\section{REFERENCES}

[1] Mengjie Xiao. The Research on the Situation and Relationship among Social Support, Resilience and Social Adjustment of Left-behind Children [D]. Guangxi Normal University, 2015. (In Chinese)

[2] Connor, K. M.,\&Davidson, J. R. T. (2003). Development of a new resilience scale: The Connor-Davidson resilience scale (CD-RISC) Depression and Anxiety, 18(2), 76-82.

[3] Comwell, B. (2003). The dynamic properties of social support: Decay, growth, and staticity, and their effects on adolescent depression. Social Forces, 81(3), 953-978.

[4] Travis Hirschi .Causes of Delinquency[M].Berkeley: Fxee Press, 2002: 16-26.

[5] Cobb S. Presidential Address-1976. Social support as moderator of life stress. Psychosom Med,1976.38(5)300- 314.

[6] Chen Chen. Preschool children's social development and parental rearing patterns [D]. Southwest University, 2012. (In Chinese) 